\title{
Reactivity of Aluminum-Functionalized Isotactic Polypropylene with Molecular Oxygen
}

\author{
Shin-ichi KоJоH, ${ }^{\dagger}$ Toshiyuki Tsutsui, Mamoru KIOKa, \\ and Norio KaSHIWA \\ Petrochemicals $R \& D$ Center, Mitsui Chemicals Incorporated, \\ 1-2 Waki 6-chome, Waki-cho, Kuga-gun, Yamaguchi 740-0061, Japan
}

(Received July 3, 1998)

\begin{abstract}
The efficiency of the synthesis of terminally hydroxylated isotactic polypropylene (i-PP) through the oxidation of Al-functionalized i-PP was evaluated and side-reactions are discussed. Al-functionalized i-PP was prepared by chain-transfer reaction by $\mathrm{Et}_{3} \mathrm{Al}$ with the $\mathrm{MgCl}_{2}-\mathrm{TiCl}_{4}$-dioctylphthalate $/ \mathrm{Et}_{3} \mathrm{Al} /$ diphenyldimethoxysilane catalyst. It was oxidized by molecular oxygen and poured into methanol for alcoholysis at chain ends. All chain-end groups of the obtained polymer were identified by ${ }^{13} \mathrm{C}$ NMR and $52 \mathrm{~mol} \%$ of Al-functionalized chain ends was found converted to hydroxyl groups. $20 \mathrm{~mol} \%$ vinylidene groups by elimination of $\beta$-hydrogen and $8 \mathrm{~mol} \%$ vinyl groups by elimination of $\beta$-methyl groups were detected. $20 \mathrm{~mol} \%$ of Al-functionalized chain ends remained unreacted. IR analysis of the resulting i-PPs showed the hydroxyl groups at $\omega$-ends to form no hydrogen bond and decrease in reaction time with molecular oxygen to lead to lower conversion to terminally hydroxylated chain ends.
\end{abstract}

KEY WORDS Polypropylene / Al-Functionalization / Chain-End Structures / Oxidation / Hydroxylation / Reactivity / Side-Reaction /

Isotactic polypropylene (i-PP) is widely used but is desired to improve properties such as adhesion and paintability to broaden fields in which i-PP is used. Terminally hydroxylated i-PP is a typical target for this purpose and oxidation of metal-functionalized i-PP is effective method for synthesizing it as follows.

i-PP having an EtZn- group at its $\omega$-end was synthesized with chain-transfer reaction by $\mathrm{Et}_{2} \mathrm{Zn}^{1}$ and terminally hydroxylated i-PP was produced through oxidation of i-PP-Zn bond with molecular oxygen. ${ }^{2}$ The efficiency of chain-transfer reactions by $\mathrm{Et}_{2} \mathrm{Zn}$ were less than $40 \%$ and oxidation efficiency of i-PP- $\mathrm{Zn}$ bonds varied widely by experimental conditions. Analysis of other chain-end groups than hydroxyl group should be conducted to determine the reasons for differences in oxidation efficiency. Predominant chain transfer by $\mathrm{Et}_{2} \mathrm{Zn}$ and ${ }^{13} \mathrm{C} \mathrm{NMR}$ of the polymer ${ }^{3}$ suggests that all Zn-functionalized chain ends in the ethanol-insoluble polymer are converted to hydroxylated chain ends and other chain ends at $1: 2$. However, the structures of other chain ends remain unclear. Terminally hydroxylated polypropylene (PP) is obtained through the oxidation of Al-functionalized PP, ${ }^{4,5}$ although most PP is atactic. In these papers, discussion on the reactivity of $\mathrm{Al}$ functionalized PP and side-reactions would be difficult, because Al-functionalization is not complete and analysis of saturated chain-end structures is absent. The reactivity of metal-functionalized i-PP with molecular oxygen and side-reactions are still unclear, although important to discuss the synthesis of terminally hydroxylated i-PP through the oxidation of i-PP-metal bonds.

Our previous paper $^{6}$ revealed that chain-transfer reaction by $\mathrm{Et}_{3} \mathrm{Al}$, which formed $\mathrm{i}-\mathrm{PP}$ having $\mathrm{Et}_{2} \mathrm{Al}$ groups at $\omega$-ends, predominantly occurred in isospesific propylene polymerization at $100^{\circ} \mathrm{C}$ with the $\mathrm{MgCl}_{2}$ $\mathrm{TiCl}_{4}$-dioctylphthalate (DOP)/Et ${ }_{3} \mathrm{Al} /$ diphenyldimethoxy- silane (DPDMS) catalyst. The i-PP is suitable for investigation of the reactivity of Al-functionalized i-PP with molecular oxygen and side-reactions, because all $\omega$-ends consist of only Al-functionalized i-butyl structure. In this paper, the synthesis of terminally hydroxylated i-PP is carried out by the oxidation of i-PP-Al bonds with molecular oxygen and subsequent alcoholysis. All chain-end groups of the resulting polymer are identified by ${ }^{13} \mathrm{C} \mathrm{NMR}$ and all reactions for the formation of the detected chain-end groups are discussed.

\section{EXPERIMENTAL}

\section{Preparation of Catalyst}

The $\mathrm{MgCl}_{2}-\mathrm{TiCl}_{4}$-DOP catalyst was prepared as follows. In a $800 \mathrm{ml}$ stainless-steel pot containing $2.8 \mathrm{~kg}$ stainless-steel balls ( $15 \mathrm{~mm}$ diameter $), 20 \mathrm{~g}(0.21 \mathrm{~mol})$ of $\mathrm{MgCl}_{2}$ were milled with $0.03 \mathrm{~mol}$ DOP for $8 \mathrm{~h}$ under nitrogen. The milled $\mathrm{MgCl}_{2}$ was treated with $200 \mathrm{ml} \mathrm{TiCl}_{4}$ at $80^{\circ} \mathrm{C}$ for $2 \mathrm{~h}$. The solid product was separated by filtration and washed twice with hexane.

\section{Propylene Polymerization}

In a 11 glass autoclave equipped with a stirrer, $500 \mathrm{ml}$ decane were added and the system was charged with propylene. $6 \mathrm{mM} \mathrm{Et}_{3} \mathrm{Al}, 0.6 \mathrm{mM}$ of DPDMS and $0.1 \mathrm{mM}$ of the solid catalyst (in term of Ti) were added at $100^{\circ} \mathrm{C}$ in this order. Polymerization was carried out under atmospheric pressure at that temperature for $1 \mathrm{~h}$. During polymerization, $501 \mathrm{~h}^{-1}$ of propylene were supplied continuously. After polymerization, the feed of the propylene monomer was stopped and replaced by a stream of nitrogen. The slurry was brought into contact with molecular oxygen.

${ }^{\dagger}$ To whom correspondence should be addressed. (Tel: +81-8275-3-9121, Fax: +81-8275-3-8891). 


\section{Hydroxylation}

Into the above slurry, $151 \mathrm{~h}^{-1}$ dry air were continuously supplied at $100^{\circ} \mathrm{C}$ for 1 or $3 \mathrm{~h}$. The whole product was poured into 41 methanol at room temperature. The slurry was stirred for $5 \mathrm{~min}$ and settled for $5.5 \mathrm{~h} .2 \mathrm{ml}$ hydrochloric acid were added to the slurry and the mixture was stirred for $5 \mathrm{~min}$, settled for $30 \mathrm{~min}$ and filtered. The polymer was washed with methanol and vacuum-dried at $60^{\circ} \mathrm{C}$ for $12 \mathrm{~h}$ (yield $1.8 \mathrm{~g}$ ).

\section{Polymer Analysis}

${ }^{13} \mathrm{C}$ NMR was performed in the following manner. A polymer solution was prepared by dissolving $150 \mathrm{mg}$ polymer sample at $120^{\circ} \mathrm{C}$ in a mixture of $0.5 \mathrm{ml}$ hexachlorobutadiene and $0.1 \mathrm{ml}$ perdeuteriobenzene. The ${ }^{13} \mathrm{C}$ NMR spectrum was recorded on a JEOL GX-500 spectrometer operated at $125.8 \mathrm{MHz}$ under proton noise decoupling in the Fourier-transform mode. Instrumental conditions were as follows: pulse angle $45^{\circ}$, pulse repetition $4.2 \mathrm{~s}$, spectral width $7500 \mathrm{~Hz}$, number of scans 20000 , temperature $110^{\circ} \mathrm{C}$, data points $64 \mathrm{~K}$.

The molecular weight of i-PP was measured by a Millipore Waters 150C gel permeation chromatograph (GPC) equipped with a refractive index detector, using a TSK mixed polystyrene gel column (G3000-G7000, exclusion limits 400000000 for polystyrene molecular weight) and $o$-dichlorobenzene as solvent at $140^{\circ} \mathrm{C}$. Number-average and weight-average molecular weights ( $M_{n}$ and $M_{w}$, respectively) were calculated on the basis of polystyrene standard calibration.

The melting temperature was measured on a PerkinElmer DSC-7 differential scanning calorimeter (DSC). First, the sample was heated to $200^{\circ} \mathrm{C}$ at $20^{\circ} \mathrm{C} \mathrm{min}^{-1}$, well above the melting temperature, and maintained at this temperature for $10 \mathrm{~min}$. Then it was cooled to $30^{\circ} \mathrm{C}$ at $10^{\circ} \mathrm{Cmin}^{-1}$ to crystallize, followed by reheating at $10^{\circ} \mathrm{C} \mathrm{min}^{-1}$. The thermogram of the sample was recorded in the second heating run to remove thermal history. The instrument was calibrated by the melting points of indium and lead.

Infrared rays analysis (IR) with the pressed sheet sample was performed. The polymer sample was heated at $180^{\circ} \mathrm{C}$ for $2.5 \mathrm{~min}$ and deaerated at this temperature with a pressing machine. It was pressed at that temperature under $7.84 \mathrm{MPa}$ for $10 \mathrm{~s}$ and cooled with another pressing machine having coolant under $9.81 \mathrm{MPa}$ for $1 \mathrm{~min}$. The obtained sheets were 0.11 and $0.12 \mathrm{~mm}$ in thickness. The IR spectrum was recorded on a JASCO A-302 spectrometer.

\section{RESULTS AND DISCUSSION}

After synthesis of Al-functionalized i-PP by the propylene polymerization with a $\mathrm{MgCl}_{2}-\mathrm{TiCl}_{4}-\mathrm{DOP} / \mathrm{Et}_{3} \mathrm{Al} /$ DPDMS catalyst system at $100^{\circ} \mathrm{C}$ for $1 \mathrm{~h}$, molecular oxygen was introduced into the polymer slurry at $100^{\circ} \mathrm{C}$ for $3 \mathrm{~h}$. The slurry was poured into excess methanol for alcoholysis at chain ends. $M_{n}, M_{w} / M_{n}$, and melting temperature of the obtained polymer were 10400, 9.3, and $155.2^{\circ} \mathrm{C}$, respectively. These values were almost the same as those ${ }^{6}$ of the i-PP prepared by the same operation except for the absence of contact with molecular oxygen. The oxidation of i-PP-Al bonds with molecular oxygen
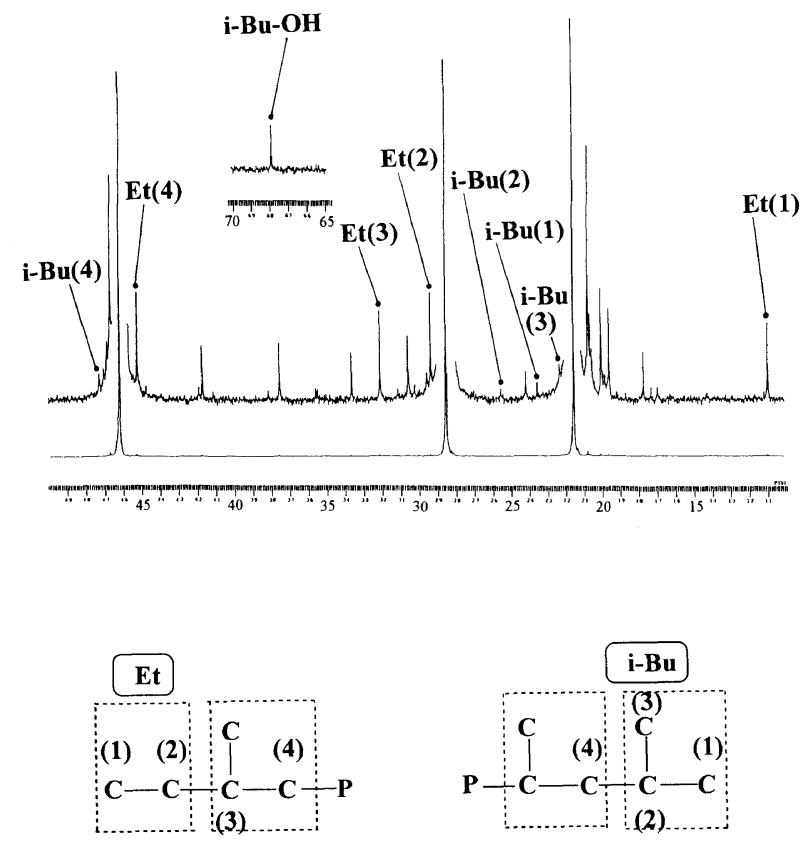

Figure 1. Saturated terminal group region of the ${ }^{13} \mathrm{C} N M R$ spectrum of hydroxylated PP polymerized at $100^{\circ} \mathrm{C}$ with the $\mathrm{MgCl}_{2}-\mathrm{TiCl}_{4}$ $\mathrm{DOP} / \mathrm{Et}_{3} \mathrm{Al} / \mathrm{DPDMS}$ catalyst.

Scheme 1 (without the molecular oxygen contact)

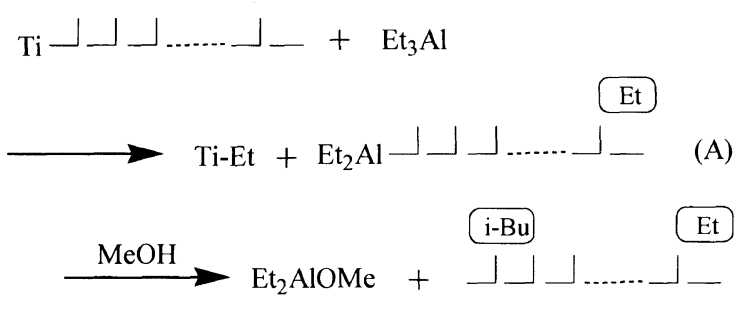

Scheme 2 (with the molecular oxygen contact)

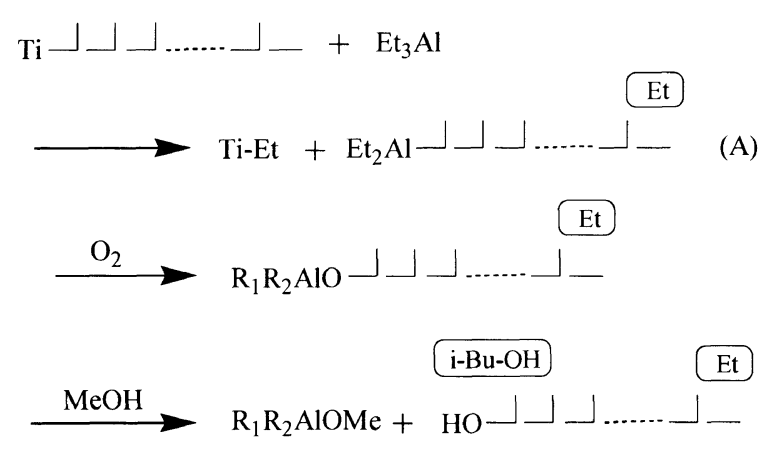

(Where $\mathrm{R}_{\mathrm{n}}$ is Et or EtO )

Figure 2. Schemes for the formation of Al-functionalized $P P$ in polymerization at $100^{\circ} \mathrm{C}$ with the $\mathrm{MgCl}_{2}-\mathrm{TiCl}_{4}-\mathrm{DOP} / \mathrm{Et}_{3} \mathrm{Al} / \mathrm{DPDMS}$ catalyst, oxidation of PP-Al bond and alcoholysis of aluminum compounds.

did not affect these values.

Figure 1 shows the saturated hydrocarbon region of the ${ }^{13} \mathrm{C}$ NMR spectrum of i-PP obtained with molecular oxygen contact. The chain-end groups of i-PP obtained 


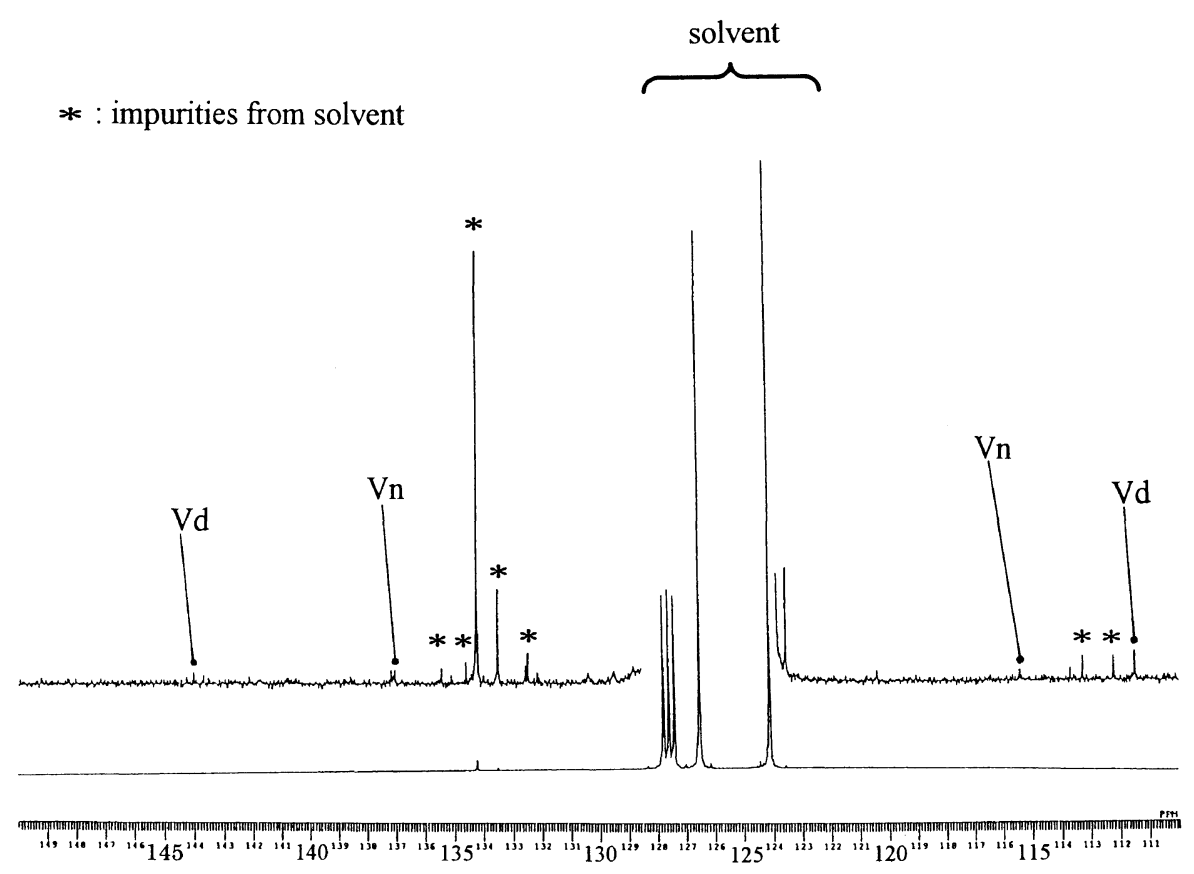

Figure 3. Unsaturated terminal group region of the ${ }^{13} \mathrm{C}$ NMR spectrum of hydroxylated $\mathrm{PP}$ polymerized at $100^{\circ} \mathrm{C}$ with the $\mathrm{MgCl}_{2}-\mathrm{TiCl}_{4}-$ $\mathrm{DOP} / \mathrm{Et}_{3} \mathrm{Al} / \mathrm{DPDMS}$ catalyst.

Table I. Proportions of chain end groups

Each value was calculated as the ratio of areas in the ${ }^{13} \mathrm{C}$ NMR spectrum; Et, average of peak areas at $11 \mathrm{ppm}$ and $32 \mathrm{ppm}$; i-Bu, average of peak areas at $23.5 \mathrm{ppm}$ and $25.5 \mathrm{ppm}$; $\mathrm{i}-\mathrm{Bu}-\mathrm{OH}$, peak area at $68 \mathrm{ppm}$; Vd, peak area at $111.5 \mathrm{ppm}$; Vn, peak area at $115.5 \mathrm{ppm}$

\begin{tabular}{|c|c|c|c|c|c|c|c|c|}
\hline \multirow{3}{*}{ Run No. } & \multirow{3}{*}{$\mathrm{O}_{2}$ treatment } & \multicolumn{7}{|c|}{ Chain end groups $/ \mathrm{mol} \%$} \\
\hline & & \multicolumn{2}{|c|}{$\alpha$-End groups } & \multicolumn{5}{|c|}{$\omega$-End groups } \\
\hline & & Et & Others & $\mathrm{i}-\mathrm{Bu}$ & $\mathrm{i}-\mathrm{Bu}-\mathrm{OH}$ & $\mathrm{Vd}$ & $\mathrm{Vn}$ & Others \\
\hline 1 & Without & 50 & N.D. ${ }^{a}$ & 50 & N.D. & N.D. & N.D. & N.D. \\
\hline 2 & With & 50 & N.D. & 10 & 26 & 10 & 4 & N.D. \\
\hline
\end{tabular}

${ }^{a}$ Not detected.

without molecular oxygen contact were found to be ethyl group (Et) and i-butyl group (i-Bu) at $1: 1,^{6}$ formed by only the reaction as shown in Scheme 1 of Figure 2. Figure 1 shows drastic decrease in the peak assigned to $\mathrm{i}-\mathrm{Bu}$ and the appearance of the peak assigned to hydroxylated i-Bu (i-Bu-OH) by molecular oxygen contact, indicating hydroxylation at the $\mathrm{Al}$-functionalized chainend group as shown in Scheme 2 of Figure 2.

The unsaturated chain-end groups shown in Figure 3 were newly detected by molecular oxygen contact and assigned to vinylidene $(\mathrm{Vd})$ and vinyl $(\mathrm{Vn})$ groups. The proportions of chain-end groups are summarized in Table I, compared with i-PP obtained without molecular oxygen contact. $26 \mathrm{~mol} \%$ of $\mathrm{i}-\mathrm{Bu}-\mathrm{OH}$ means that 52 mol $\%$ of obtained i-PP was terminally hydroxylated i-PP. $10 \mathrm{~mol} \%$ of $\mathrm{i}-\mathrm{Bu}$ in Table I means the presence of $20 \mathrm{~mol} \%$ of $\mathrm{Al}$-functionalized i-PP which remained unreacted with molecular oxygen. Zn-functionalized i-PP did not produce $\mathrm{i}-\mathrm{Bu}^{3}$, although it contained unknown chain ends. Higher stability of Al-functionalized i-PP during molecular oxygen contact may thus be indicated. The difference between $M_{n}$ of Al-functionalized i-PP, which was 10400 , and that of $\mathrm{Zn}$-functionalized i-PP ${ }^{3}$, 3300 , permits us to interpret the higher stability of Al-functionalized i-PP in two ways. One is that the
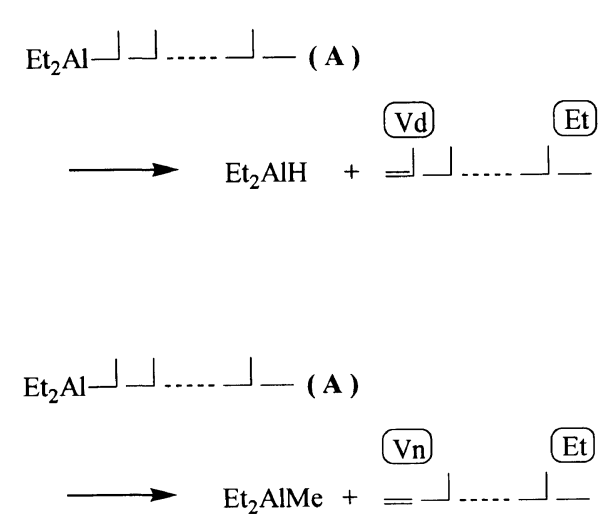

Figure 4. Schemes for the formation of unsaturated chain-end groups due to decomposition of Al-functionalized PP.

bulkier metal-functionalized i-PP showing higher $M_{n}$ has lower reactivity with molecular oxygen due to steric hindrance. The other is that the higher molecular weight means lower reactivity of $\mathrm{Al}$ compound as a chain transfer reagent and suggests the lower reactivity of Al-functionalized chain ends during molecular oxygen contact.

The presence of $\mathrm{Vd}$ may show the decomposition of Al-functionalized i-PP by the elimination of $\beta$-hydrogen 


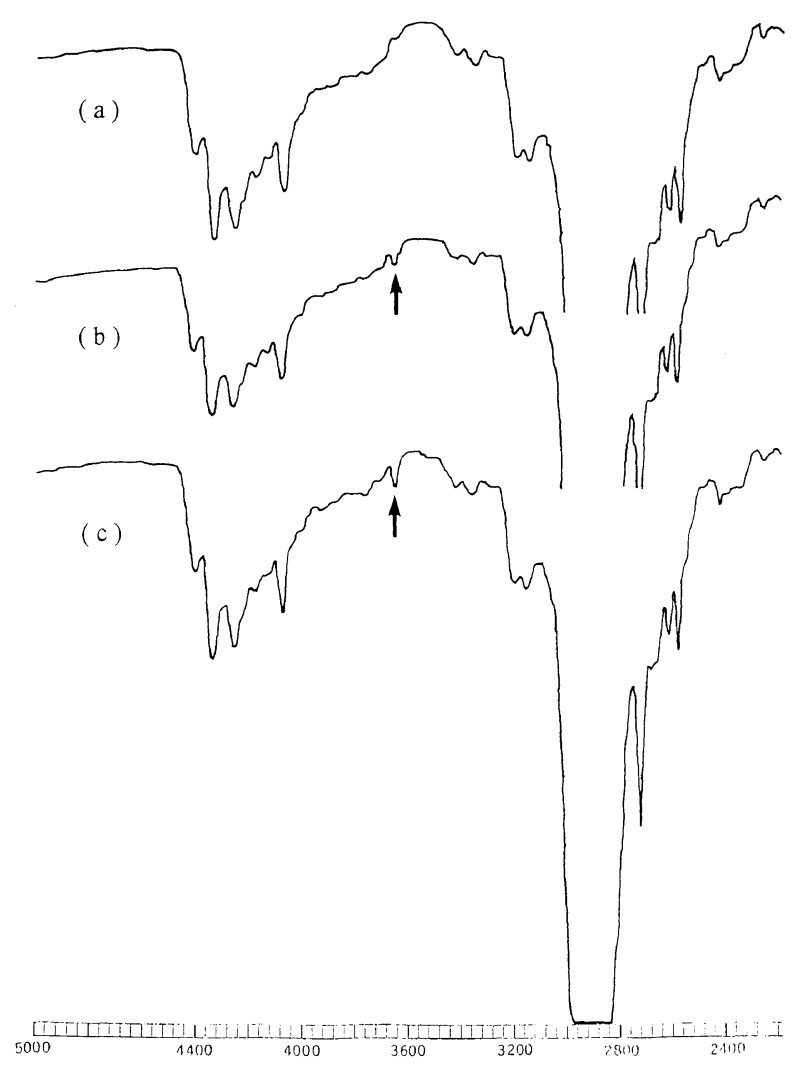

Figure 5. IR spectra of PPs prepared with and without molecular oxygen contact: (a) without molecular oxygen contact; (b) with the molecular oxygen contact for $1 \mathrm{~h}$; (c) for $3 \mathrm{~h}$.

as shown in Scheme (3) of Figure 4. The detection of $10 \mathrm{~mol} \%$ of $\mathrm{Vd}$ suggests that $20 \mathrm{~mol} \%$ of $\beta$-hydrogen of Al-functionalized i-PP was possibly eliminated. Such decomposition of aluminum compound at high temperature is well-known. ${ }^{7}$

A plausible interpretation of the presence of $\mathrm{Vn}$ is that the 2,1-insertion of propylene monomer occurs followed by $\beta$-hydrogen elimination of the methyl group. ${ }^{5}$ However, such chain-transfer reaction does not occur under the present polymerization conditions. ${ }^{6}$ The detection of $4 \mathrm{~mol} \%$ of $\mathrm{Vn}$ would be explained by the finding that $8 \mathrm{~mol} \%$ of $\beta$-methyl group of Al-functionalized i-PP may be eliminated as shown in Scheme (4) of Figure 4. In previous papers, ${ }^{8,9}$ the elimination of $\beta$-methyl group of $\mathrm{Zr}$-functionalized i-PP was noted in propylene polymerization at high temperature with the zirconocene/methylaluminoxian catalyst.

Figure 5 shows the IR spectra of pressed sheets of i-PPs prepared with molecular oxygen contact for 0,1 , and $3 \mathrm{~h}$, respectively. By contact with molecular oxygen, the peak at $3650 \mathrm{~cm}^{-1}$ was newly detected. This peak was assigned to stretching vibrations of $\mathrm{O}-\mathrm{H}$ bonds which do not form hydrogen bonds. The peak for the sample prepared with molecular oxygen contact for $3 \mathrm{~h}$ was stronger than for $1 \mathrm{~h}$, indicating longer time of contact with molecular oxygen to possibly increase the oxidation of i-PP-Al bonds. The absence of hydrogen bonds formed by i-Bu-OH would indicate high dispersion of hydroxyl groups in the film. It would be favorable for improving the properties of i-PP by using hydroxyl groups.

This paper presents an effective method to synthesize terminally hydroxylated i-PP of high molecular weight. This method enables us to discuss the reactivity of $\mathrm{i}$ PP-Al bonds with molecular oxygen and side-reactions. Although it takes more time to estimate how terminally hydroxylated i-PP improves the properties of i-PP such as adhesion or paintability, the results in this paper show reason for further investigation.

\section{REFERENCES}

1. J. J. Boor, J. Polym. Sci., Part C, 1, 237 (1963).

2. D. R. Burfield, Polymer, 25, 1817 (1984).

3. T. Shiono, K. Yoshida, and K. Soga, Makromol. Chem., Rapid Commun., 11, 169 (1990).

4. K. K. Kang, T. Shiono, and T. Ikeda, Macromolecules, 30, 1231 (1997).

5. T. Shiono, K. K. Kang, H. Hagihara, and T. Ikeda, Macromolecules, 30, 5997 (1997).

6. S. Kojoh, M. Kioka, N. Kashiwa, M. Itoh, and A. Mizuno, Polymer, 36, 5015 (1995).

7. K. Ziegler, H. G. Gellert, H. Lehmkuhl, W. Pfohl, and K. Zoesel, Ann. Chem., 1, 629 (1960).

8. J. Eshuis, Y. Tan, A. Meetsma, and J. H. Teuben, Organometallics, 11, 362 (1992).

9. L. Resconi, F. Piemontesi, I. Camurati, O. Sudmeijer, I. E. Nifant'ev, P. V. Ivchenko, and L. G. Kuz'mina, J. Am. Chem. Soc., 120, 2308 (1998). 